\title{
TITLE:
}

\section{Defect characteristics by boron cluster ion implantation}

$\operatorname{AUTHOR}(\mathrm{S})$ :

Aoki, Takaaki; Matsuo, Jiro; Takaoka, Gikan; Toyoda, Noriaki; Yamada, Isao

\section{CITATION:}

Aoki, Takaaki ...[et al]. Defect characteristics by boron cluster ion implantation. Nuclear Instruments and Methods in Physics Research Section B: Beam Interactions with

Materials and Atoms 2003, 206: 855-860

\section{ISSUE DATE:}

2003-05

URL:

http://hdl.handle.net/2433/8943

\section{RIGHT:}

This is not the published version. Please cite only the published version.; この論文は出版社版でありません。引用の際には出版社版を ご確認ご利用ください。 
Defect Characteristics by Boron Cluster Ion Implantation

Takaaki Aoki $^{1,3)}$, Jiro Matsuo ${ }^{1)}$, Gikan Takaoka ${ }^{1)}$, Noriaki Toyoda ${ }^{2)}$, Isao Yamada ${ }^{2,3)}$

1) Ion Beam Engineering Experimental Laboratory, Kyoto University, Sakyo, Kyoto 606-8501, JAPAN

2) Laboratory of Advanced Science and Technology for Industry, Himeji Institute of Technology, CAST, 3-1-2 Kouto, Kamigori-cho, Ako-gun, Hyogo, 678-1205 JAPAN

3) Collaborative Research Center for Cluster Ion Beam Process Technology.

Corresponding Author:

Takaaki Aoki

Ion Beam Engineering Experimental Laboratory, Kyoto University

Sakyo Kyoto, 606-8501, JAPAN

TEL: +81-75-753-4994 / FAX: +81-75-751-6774

e-mail: t-aoki@kuee.kyoto-u.ac.jp

\section{$\underline{\text { ABSTRACT }}$}

Cluster ion implantation using decaborane $\left(\mathrm{B}_{10} \mathrm{H}_{14}\right)$ has been proposed as a shallow implantation technique for LSI devices with gate lengths of several-tens nanometers. Experiments and computer simulations of low-energy boron monomers and decaborane clusters implantation were performed. Molecular dynamics simulations of $\mathrm{B}_{10}$ cluster implantation have 
shown similar implant depth but different damage density and damage structure compared to monomer $\left(\mathrm{B}_{1}\right)$ ion implantation with the same energy-per-atom. For monomer implantation, point-defects such as vacancy-interstitial pairs are mainly formed. On the other hand, $\mathrm{B}_{10}$ generates large numbers of defects within a highly-amorphised region at the impact location. This difference in damage structure produced during implantation is expected to cause different annihilation processes.

PACS:

61.80.Lj (Atom and molecule irradiation effects)

36.40.-c (Atomic and molecular clusters)

61.72.Ji (Point defects and defect clusters)

\section{INTRODUCTION}

As the scale of LSI device decreases, the formation of ultra shallow p-type junctions becomes more important. Cluster ion implantation using small boron cluster, decaborane $\left(\mathrm{B}_{10} \mathrm{H}_{14}\right)$, has been introduced as a candidate for ultra shallow junction formation. Both experiments [1,2] and molecular dynamics (MD) simulations [3] of small B cluster and monomer implantation have been performed. From previous work [3], it has been reported that, when a B cluster with size below 10 and energy of several hundreds $\mathrm{eV}$ per atom impacts on Si substrate, the cluster breaks up in the substrate and each B atom penetrates independently into the substrate. Therefore, the depth profile of B atoms by cluster implantation was the 
same as that by B monomer ions at the same energy per atom.

Additionally, it is important to understand the damage formation process because the annealing after ion implantation causes transient enhanced diffusion (TED) of dopant $[4,5]$, which is one of the problems in fabrication of high-quality shallow junctions. It has been considered that the TED has close relation to the structure of damage formed by ion implantation. It is necessary to discuss the similarities and differences between monomer and cluster implantation processes.

In this paper, $\mathrm{MD}$ simulations of $\mathrm{B}_{1}$ monomer and $\mathrm{B}_{10}$ cluster impacting on a $\mathrm{Si}(100)$ substrate are discussed. The defects induced by ion impact are classified into vacancies and interstitials. The distribution and structures of these defects are examined. The differences of damage formation and annihilation mechanism between monomer and cluster impact are discussed. Defect formation processes were examined experimentally using the RBS channeling method. By both experimental and simulation methods, the damage accumulation processes by boron monomer and cluster implantations are discussed.

\section{$\underline{\text { SIMULATION MODEL }}$}

In order to examine the process of damage formation by small boron cluster impacts, MD simulations of $\mathrm{B}_{1}$ and $\mathrm{B}_{10}$ monomer/cluster impact on a $\mathrm{Si}(100)$ substrate were performed. The Stillinger-Weber potential model [6] was applied to the inter-atomic potential of Si-Si, and the Ziegler, Biersack, and Littmark (ZBL) model [7] was applied to B-B and B-Si. A $\operatorname{Si}(100)$ substrate consists of 32768 atoms with a cube side of about $90 \AA$ was prepared as a target material. The periodic boundary condition was applied on the lateral directions of this 
target. The some bottom layer atoms were fixed to keep bulk structure. The other side, where B monomer or cluster impacts, was kept in free boundary.

$\mathrm{B}_{1}$ and $\mathrm{B}_{10}$ are radiated on the $\mathrm{Si}$ substrate. $\mathrm{B}_{1}$ monomers were implanted with an incident angle of $7^{\circ}$ to the surface normal and rotated $30^{\circ}$ to the (100) direction to avoid channeling implantation. $\mathrm{B}_{10}$ has spherical structure and was implanted at normal direction to the surface. In previous work [3], it was confirmed that a spherical $\mathrm{B}_{10}$ cluster shows similar penetration depth and implant efficiency to monomer ions with same incident energy per atom. In order to obtain statistical properties, 50 simulations for $\mathrm{B}_{1}$ and 25 simulations for $\mathrm{B}_{10}$ were performed respectively at different impact points. The simulation time was 8ps. During this time, the incident B atom loses its kinetic energy and stops in the target. This time is also enough long that transient displacements by ion impact recovers. Displacements produced by ion impact were investigated after the model by Pérez-Martín et al. [8]. A vacancy is defined as a lattice point that has no atoms bound within $1.6 \AA$, and an interstitial is an atom that is father than $1.6 \AA$ from any lattice points.

\section{RESULTS AND DISCUSSION}

Figure 1 shows snapshots of incident B atoms, interstitials and bonded Si lattice atoms during the impacts of $\mathrm{B}_{1}$ and $\mathrm{B}_{10}, 8 \mathrm{ps}$ after impact. Incident energy is $230 \mathrm{eV} /$ atom. The cut-off radius to select a surrounding Si atom has been set to $2.7 \AA$, which is between the first and the second nearest neighbor length in the Si crystal. The incident B atoms are indicated with circles (in the left snapshot of $B_{1}, B$ is backscattered). At the impact of $B_{1}$, interstitial 
atoms are separated from each other or remain as small interstitial clusters, such as $I_{2}$ structure. On the other hand, at the impact of $\mathrm{B}_{10}$, most of interstitial atoms are found in the near surface region of the impact point. Additionally, separated interstitials, which are mainly shown for $\mathrm{B}_{1}$ impact, are formed far from the surface, in other words, at 'end-of-range.' The incident B atoms are distributed over wider range compared with the range where the high-density interstitials reside.

Figure 2 shows depth distributions of vacancies and interstitials by $\mathrm{B}_{1}$ and $\mathrm{B}_{10}$ implantation. For $\mathrm{B}_{10}$, the profiles have been normalized to indicate the number of vacancies or interstitials by one B atom in a cluster. These profiles are similar to the depth profiles of damage modeled by M. J. Catula et al. [9], where a displacement is defined as a Si atom which has a potential energy of $0.4 \mathrm{eV}$ above the bulk state [3]. As shown in figure 2, the depth ranges of vacancies and interstitials by $\mathrm{B}_{1}$ and $\mathrm{B}_{10}$ are similar to each other. However, $\mathrm{B}_{10}$ impact causes very high-density of defects in the near surface region ranging from the surface to the depth of $20 \AA$. This distribution is comparable to the mean implant depth of B monomers and clusters [3]. Considering the density of interstitials shown in figure $2, \mathrm{~B}_{10}$ impact causes about 15 interstitials per $2 \AA$ thickness. From these results, it is expected that the impact point is completely amorphised by only one $\mathrm{B}_{10}$ ion impact.

Figure 3 shows radial distributions of substrate Si atoms around vacancies and interstitials. In the case of $B_{1}$ impact, the distribution around vacancies shows distinct peaks and valleys. Compared with the radial distribution function of bulk Si crystal, each peak in $\mathrm{B}_{1}$ distribution shifts to nearer side, but shows good correlation with one in Si crystal. As for the 
distribution around interstitials at $B_{1}$ impact, the second peak is observed at $3.1 \AA$ with similar intensity to the first-nearest peak. Those two distributions indicate that, at the impact of $\mathrm{B}_{1}$, only one knocked-on atom is displaced from its lattice site but other surrounding atoms maintain lattice structure, which results in the formation of point-defects with certain structures $[8]$.

Following the impact of $\mathrm{B}_{10}$, the peak-and-valley structure is not found around vacancies within $3 \AA$. This result means that the lattice structures before and after impact have no correlation with each other; in other words, damage structure can be fully amorphised. The distribution around interstitials also becomes broad as the distance increases. From these results, it can be concluded that the implant region is fully amorphised by only one $\mathrm{B}_{10}$ impact at $230 \mathrm{eV} /$ atom. As reported in the previous works [4,5], it is known that TED can be reduced by pre-amorphisation of the surface region to sufficient depth compared to the boron implant range. Thus, characteristic damage formation by $\mathrm{B}_{10}$ is expected to result in advantages for shallow junction formation.

As the ion dose increases, the damage in the target is accumulated. When the ion dose is enough large, the implanted region becomes fully amorphised. The damage accumulation process has been examined by both simulations and experiments. Figure 4 shows the MD simulation results of the evolutions of interstitials with sequential irradiation of $\mathrm{B}_{1}$ and $\mathrm{B}_{10}$ at energy of 200 or $500 \mathrm{eV} /$ atom. The $\mathrm{x}$-axis represents the number of irradiated B atoms. In both cases of 200 and $500 \mathrm{eV} /$ atom, $\mathrm{B}_{10}$ implantation shows rapid growth of defects compared to $\mathrm{B}_{1}$, and moreover, the surface is completely amorphised at atomic dose of $1 \times 10^{15} / \mathrm{cm}^{2}$ 
$\left(1 \times 10^{14} / \mathrm{cm}^{2}\right.$ in ion dose). Similar examination was carried out experimentally. B monomer and decaborane $\left(\mathrm{B}_{10} \mathrm{H}_{14}\right)$ were radiated on $\mathrm{Si}(100)$ surface with $500 \mathrm{eV} /$ atom and damage was measured by RBS channeling. Figure 5 shows the boron atomic dose dependence of the number of displacements by $\mathrm{B}$ and $\mathrm{B}_{10} \mathrm{H}_{14}$ implantation obtained by RBS experiments. Comparing experimental and simulation results, both curves show good quantitative agreement with each other.

\section{SUMMARY}

Molecular dynamics simulations of impacts by boron monomers and small boron clusters were performed and the damage caused by these ion irradiations has been investigated.

For $\mathrm{B}_{1}$ implantation, the damage is mainly due to formation of vacancy-interstitial pairs, where one lattice Si atom is knocked-on and surrounding Si atoms keep lattice structure. The knocked-on atom tends to remain as a tetrafold interstitial. However, a $\mathrm{B}_{10}$ cluster creates a high density of displacements around the impact point because of the high-density energy irradiation effect. This damaged region is considered to be amorphised and appears as a box-like shape from the surface to a depth of $20 \AA$, which is comparable with the mean implant depth of the B atoms.

The damage accumulation process was studied by both experimental and simulation methods. At incident energy of $500 \mathrm{eV}$, simulation and experimental results have shown good agreement quantitatively. Both results showed that, at $\mathrm{B}_{10}$ implantation with $500 \mathrm{eV} /$ atom, the number of displacements increases more rapidly compared to $\mathrm{B}_{1}$ impact, and the surface is 
well amorphised at the atomic dose of $1 \times 10^{15} / \mathrm{cm}^{2}$.

These results support the availability of decaborane ion implantation as a low-energy boron implantation technique for LSI fabrication.

\section{ACKNOWLEDGEMENT}

The authors thankfully acknowledge NEDO (New energy and industrial technology development organization) in Japan for supporting this work.

\section{REFERENCES}

[1] K. Goto, J. Matsuo, T. Sugii, H. Minakata, I. Yamada and T. Hisatsugu, IEDM Tech. Digst., 435 (1996).

[2] K. Goto, J. Matsuo, Y. Tada, Y. Momiyama, T. Sugii and I. Yamada, IEDM Tech. Digst., 471 (1997).

[3] T. Aoki, J. Matsuo, Z. Insepov and I. Yamada, 1998 International Conference on Ion Implantation Technology Proceedings, 1254 (1999).

[4] D. J. Eaglesham, P. A. Stolk, H. J. Gossman and J. M. Poate, Appl. Phys. Lett., 65, 2305 (1994).

[5] K. S. Jones, P. G. Elliman, M. M. Petravic, and P. Kringhoj, Appl. Phys. Lett., 68, 3111 (1996).

[6] F. H. Stillinger and T. A. Weber, Phys. Rev., B31, 5632 (1985). 
[7] J. P. Ziegler, J. P. Biersack and U. Littmark, The stopping and range of ions in solids; New York: Pergamon Press (1985).

[8] A. M. C. Pérez-Martín, J. Domínguez-Vázquez, J. J. Jiménez-Rodoríguez, Nucl. Instr. and Meth., B164-165, 431 (2000).

[9] M. J. Caturla, T. D. de la Rubia and G. H. Gilmer, Nucl. Instr. and Meth. B106, 1 (1995).
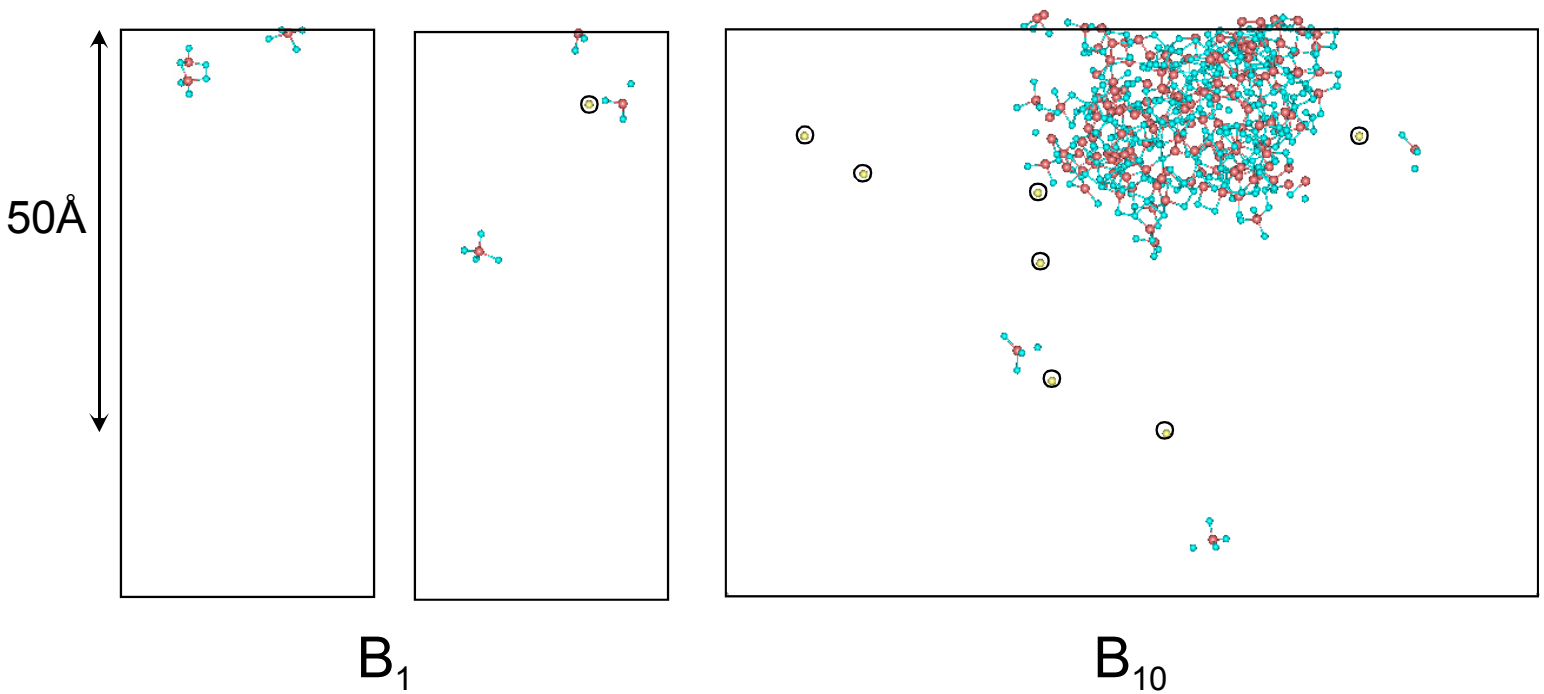

Figure 1: Snapshots of interstitial and bonded lattice Si implanted with $\mathrm{B}_{1}$ and $\mathrm{B}_{10}$. Incident $\mathrm{B}$ atoms are indicated with circles 


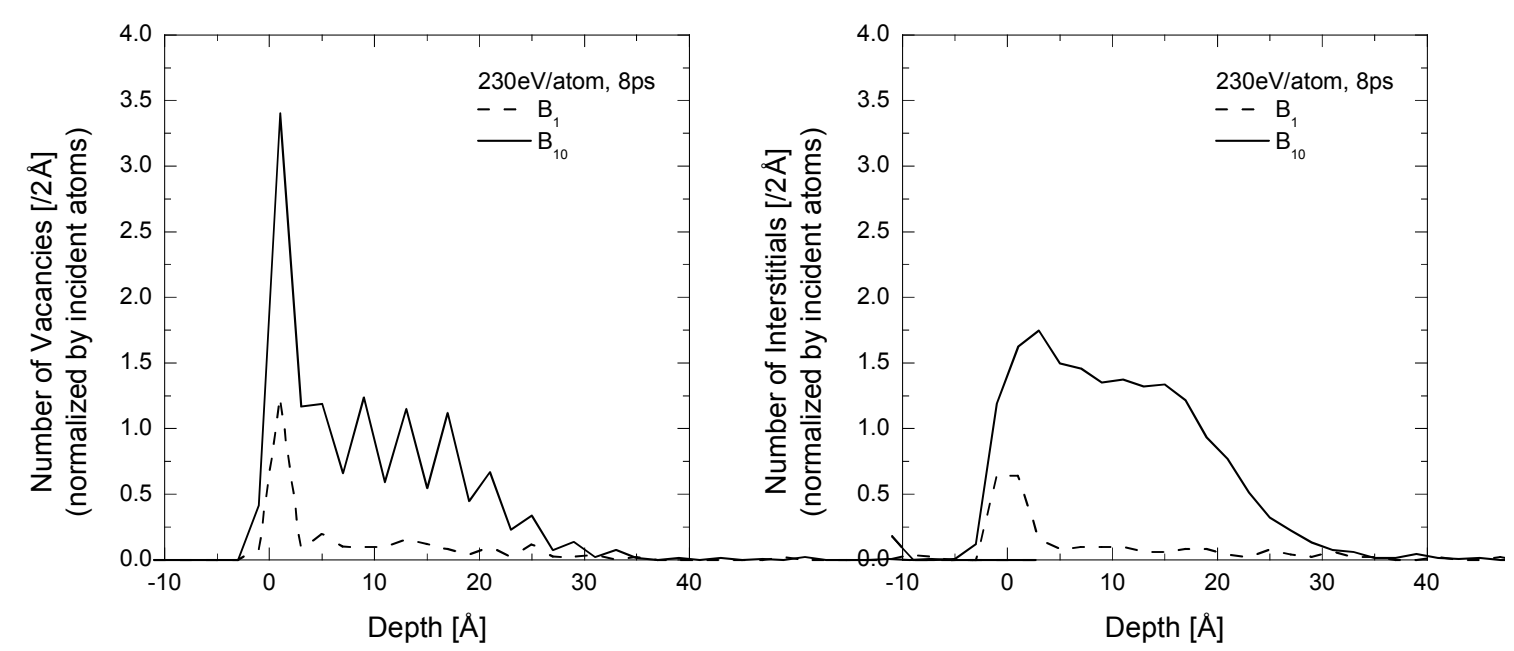

Figure 2: Depth profile of vacancies and interstitials by B monomer and cluster implantation.
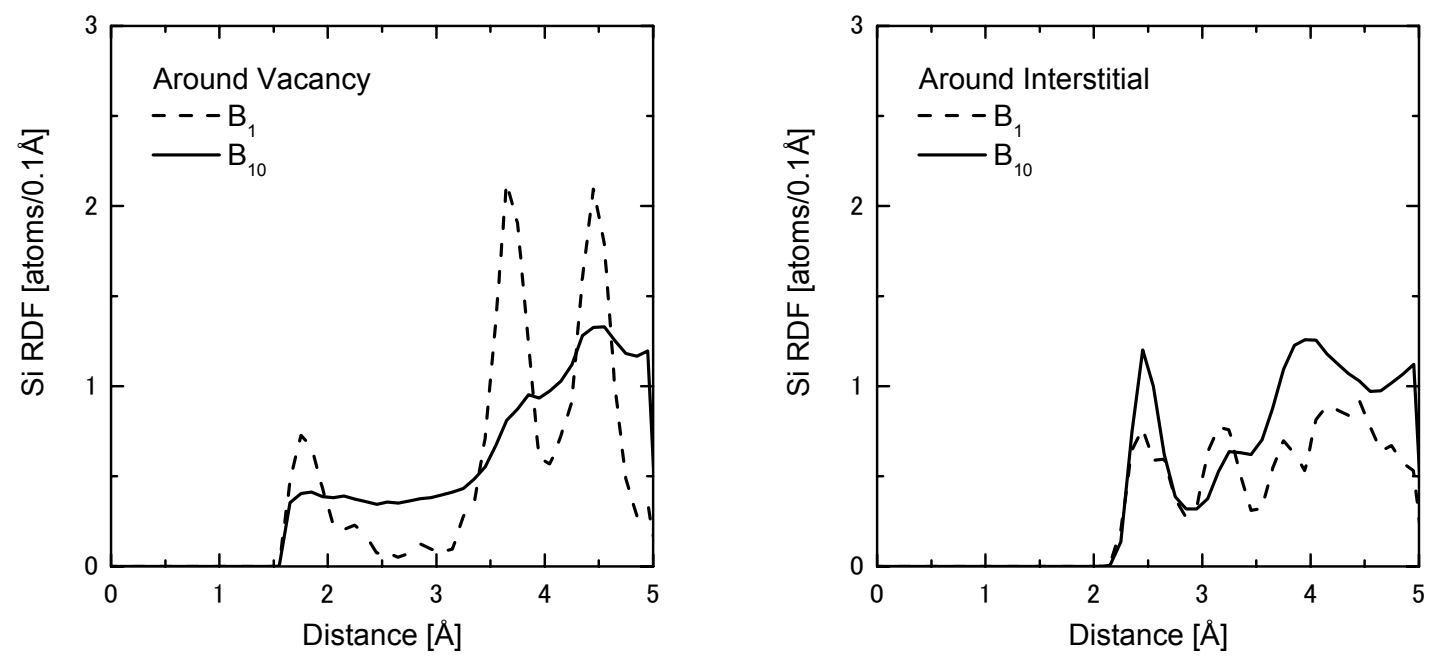

Figure 3: Radial distribution of substrate Si atoms around vacancies and interstitials. 

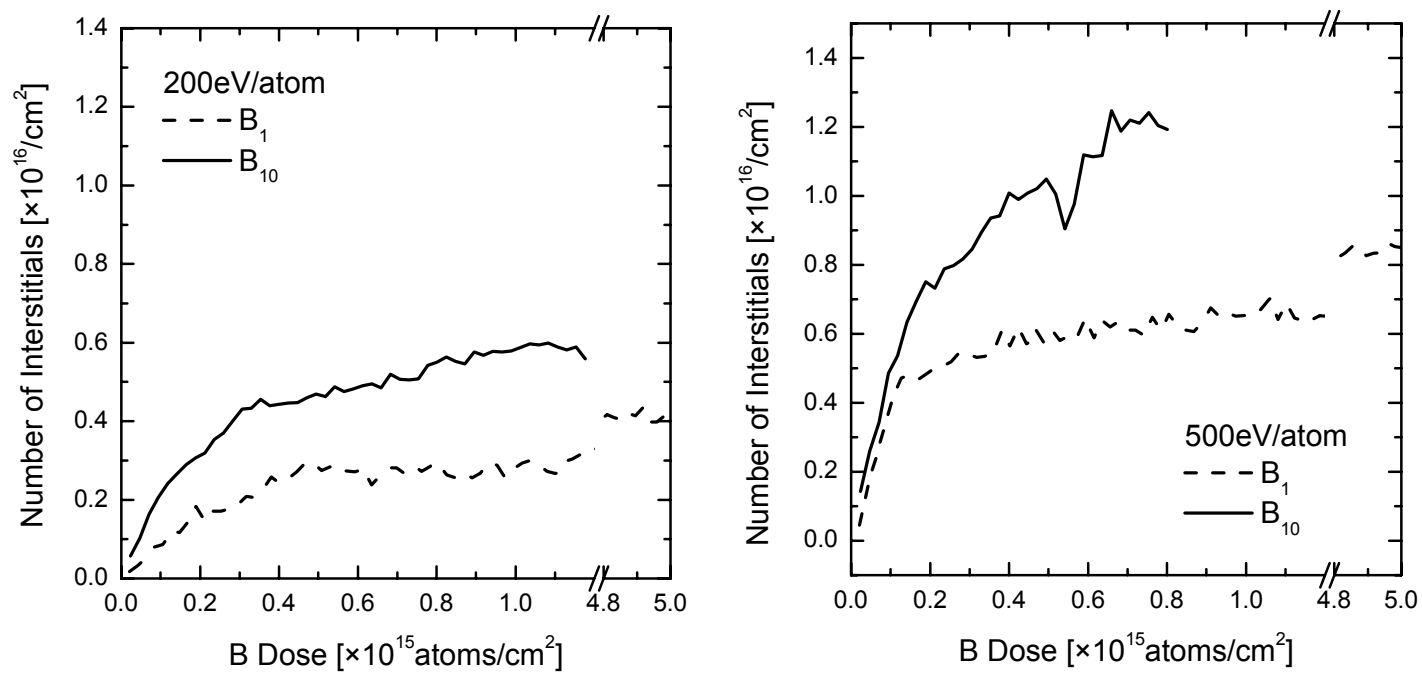

Figure 4: B atomic dose dependence of number of interstitials induced by B monomer and cluster, simulation result.

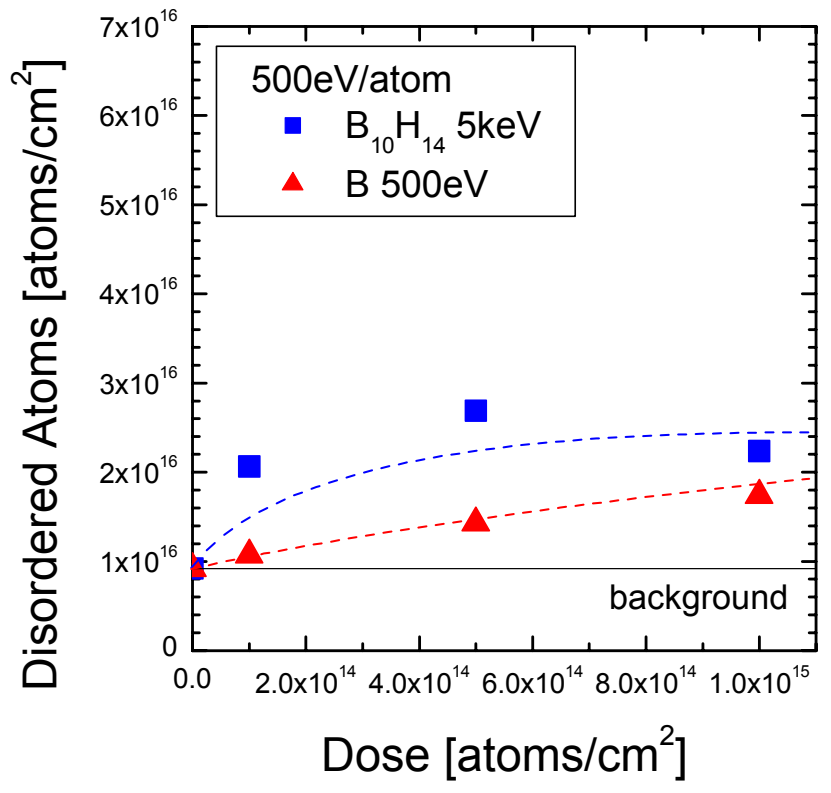

Figure 5: B atomic dose dependence of number of interstitials induced by B monomer and cluster, experimental result. 